\title{
New R Coronae Borealis stars discovered in OGLE-III Galactic bulge fields from their mid- and near-infrared properties
}

\author{
P. Tisserand ${ }^{1}$, L. Wyrzykowski ${ }^{2,3}$, P. R. Wood ${ }^{1}$, A. Udalski ${ }^{3}$, M. K. Szymański ${ }^{3}$, M. Kubiak ${ }^{3}$, G. Pietrzyński ${ }^{3,4}$, \\ I. Soszyński ${ }^{3}$, O. Szewczyk ${ }^{3,4}$, K. Ulaczyk ${ }^{3}$, and R. Poleski ${ }^{3}$ \\ ${ }^{1}$ Research School of Astronomy and Astrophysics, Australian National University, Cotter Rd, Weston Creek ACT 2611, Australia \\ e-mail: tisserand@mso.anu.edu.au \\ 2 Institute of Astronomy, University of Cambridge, Madingley Road, Cambridge CB3 OHA, England \\ 3 Warsaw University Astronomical Observatory, Al. Ujazdowskie 4, 00-478 Warszawa, Poland \\ ${ }^{4}$ Universidad de Concepción, Departamento de Fisica, Astronomy Group, Casilla 160-C, Concepción, Chile
}

Received 9 February 2010 / Accepted 22 June 2010

\section{ABSTRACT}

\begin{abstract}
Context. An R Coronae Borealis (RCB) star is a rare type of supergiant star that is increasingly suspected to be the evolved merger product of two white dwarfs. Lately, many RCBs have been found distributed in a thin disk structure embedded inside the Galactic bulge. This unexpectedly high density may provide additional insight into the nature and age of RCB stars.

Aims. We apply and test a new technique to find RCB stars based on their particular infrared emission due to circumstellar shell. We attempt to demonstrate that RCB stars can be identified without performing a light curve analysis, which would simplified the search outside optically monitored fields.

Methods. We selected RCB candidates based on their near-infrared excess and on their particular mid-infrared shells emission, using photometric data from the 2MASS and Spitzer/GLIMPSE surveys. Each candidates OGLE light curves were then visually inspected and we selected for spectroscopy follow-up those that underwent large and rapid declines.

Results. We discover two new R Coronae Borealis stars, but also indicate four new possible candidates. We emphasize that all of the 7 known RCB stars located in both the Spitzer/GLIMPSE and OGLE-III fields were also re-discovered, which illustrates the high efficiency of our analysis.

Conclusions. The proposed new technique to find RCB stars has been successful. It can now be extended to larger areas, in particular where the interstellar extinction is too high to be monitored by optical microlensing surveys, such as the inner part of the Galactic bulge.
\end{abstract}

Key words. stars: carbon - stars: AGB and post-AGB - Galaxy: bulge - supergiants

\section{Introduction}

$\mathrm{R}$ Coronae Borealis star (RCB) is a rare type of evolved $\mathrm{H}$-deficient and carbon-rich supergiant star that is increasingly suspected to result from the merger of two white dwarfs (one $\mathrm{CO}$ - and one He-), called the Double Degenerate (DD) scenario. The DD model has been strongly supported by two observations: an ${ }^{18} \mathrm{O}$ over abundance in seven $\mathrm{H}$-deficient carbon and cool RCB stars (Clayton et al. 2007), and the large abundances of fluorine found in the hotter RCB atmospheres (Pandey et al. 2008). In this scenario, observations of RCB stars would not only help us to constrain simulations of low mass Double Degenerate merging events (Fryer \& Diehl 2008; Diehl et al. 2008), but RCB birthrates would also help us to constrain the rate of white dwarf mergers that could become, at higher mass, supernovae type Ia progenitors (Webbink 1984; Belczynski et al. 2005).

$\mathrm{R} \mathrm{CrB}$ is the prototype RCB star. Over the past $\sim 200$ years, it has displayed unpredictably fast declines in brightness, up to $9 \mathrm{mag}$ in the visible. These declines are the main signature of RCB type stars. They are caused by newly formed dust clouds that shadow their central stars. These clouds, consisting of amorphous carbon, are understood to be formed near the stellar atmo- sphere and accelerated away by radiation pressure. A detailed review of RCB characteristics was given by Clayton (1996).

RCB stars in the Large Magellanic Cloud are distributed mostly along the bar (Alcock et al. 2001; Tisserand et al. 2009; Soszyński et al. 2009). Reciprocally, the majority of Galactic RCB stars seem to be concentrated in the bulge. Indeed, a high number of RCB stars (13) have been found inside the Galactic bulge (Tisserand et al. 2008) with the surprising peculiarity of being distributed in a thin disk structure. These new discoveries indicate that the density of these rare stars is expected to be high at low Galactic latitude (more than 1 per $\operatorname{deg}^{2}$ at $|b| \leqslant 1 \mathrm{deg}$ ).

It is necessary to increase the number of known bulge RCB stars to understand their spatial distribution and therefore constrain their age and past evolution. However this research is difficult in this particular area of the sky because of the high interstellar extinction and the very high density of stars. We note that with an RCB phase lifetime of about $10^{5}$ years, as predicted by theoretical evolution models (Saio \& Jeffery 2002), and an estimated He-CO white dwarfs merger birthrate between $\sim 10^{-3}$ and $\sim 5 \times 10^{-3}$ per year (Nelemans et al. 2001; Ruiter et al. 2009), we can expect between 100 and $500 \mathrm{RCB}$ stars to exist in our Galaxy. We actually know about 50 of them (see Tisserand et al. 2008 , and references therein), which is only a factor of two more 
than the Magellanic Clouds, where 23 RCBs are known (Alcock et al. 2001; Tisserand et al. 2004, 2009).

In this article, we present a new method to search for RCB stars at low Galactic latitude in the OGLE-III fields. This analysis uses pragmatic criteria and should be considered as a test before extending an RCB search to the entire bulge area. We used the RCB stars peculiar circumstellar shell emission (Tisserand et al. 2009) to reduce the number of preselected candidates for further study, instead of highly time-consuming classical methods based on optical light curve analysis. Thanks to the publically available mid-infrared Spitzer/GLIMPSE and near-infrared 2MASS databases, only a few hundred objects were preselected before visual inspection of their OGLE-II and -III light curves.

This article reports on the analysis and discovery of two new RCB stars and four new candidates. The photometric and spectroscopic data used are presented in Sect. 2 and the detection techniques in Sect. 3. The general characteristics of the newly discovered RCBs are discussed in Sect. 4.

\section{Observational data}

Our analysis used photometric data from four different surveys: 2MASS, Spitzer/GLIMPSE, OGLE-II/III, and EROS-2. The last two projects monitored millions of stars distributed over about one hundred square degrees in the Magellanic Clouds and the Galactic bulge to search primarily for microlensing events produced by potential dark matter candidates in the halo (Tisserand et al. 2007; Wyrzykowski et al. 2009) or to ordinary stars in the Galactic plane (Sumi et al. 2006; Hamadache et al. 2006). The OGLE (Optical Gravitational Lensing Experiment) survey has known multiple phases. In this article, we used data from the second, OGLE-II (Szymanski 2005), and third generation OGLE-III (Udalski 2003). OGLE-III started in June 2001 and continued observing until May 2009, when it was upgraded to OGLE-IV. It used the 1.3-m Warsaw telescope located at Las Campanas Observatory, Chile, and a $35 \times 35.5$ arcmin field of view camera that used eight SITe $2048 \times 4096$ CCD detectors with $15 \mu \mathrm{m}$ pixels resulting in a 0.26 arcsec/pixel scale. The OGLE observations were taken mostly in $I$ band, but with occasional $V$ band exposures. The EROS-2 (Experience de Recherche d'Objets Sombres) experiment performed observations between July 1996 and February 2003 using the 1-m MARLY telescope at ESO La Silla Observatory, Chile. Two wide-field cameras of $\sim 0.95 \mathrm{deg}^{2}$ field of view were operated and placed behind a dichroic cube, which split the light beam into two broad passbands ( $B_{\mathrm{e}}$ and $R_{\mathrm{e}}$ ). Each camera had a mosaic of eight $2048 \times 2048$ CCDs with a pixel size of $0.6^{\prime \prime}$ on the sky.

The near- and mid- infrared photometric data were obtained, respectively, from the 2MASS (Skrutskie et al. 2006) and Spitzer/GLIMPSE (Churchwell et al. 2009) surveys. 2MASS used two highly-automated $1.3-\mathrm{m}$ telescopes, one at Mt. Hopkins, AZ, and one at CTIO, Chile. Each telescope was equipped with a three-channel camera, each channel consisting of a $256 \times 256$ array of $\mathrm{HgCdTe}$ detectors, capable of observing the sky simultaneously at $J$ (1.25 microns), $H$ (1.65 microns), and $K$ (2.17 microns). GLIMPSE consists of three separate surveys that observed the Galactic disk and bulge $(|b| \leq 4.5 \mathrm{deg})$ at wavelengths 3.6, 4.5, 5.8, and $8.0 \mu \mathrm{m}$ using the Infrared Array Camera IRAC (Fazio et al. 2004) on board of the Spitzer satellite.

Spectroscopy of RCB candidates was performed with the Wide Field Spectrograph (WiFeS) instrument (Dopita et al.
2007) attached to the $\mathrm{ANU}^{1} 2.3 \mathrm{~m}$ diameter telescope at Siding Spring Observatory. WiFes is an integral field spectrograph permanently mounted at the Nasmyth A focus. It provides a $25 \times 38$ arcsec field with 0.5 arcsec sampling along each of twenty five $38 \times 1$ arcsec slitlets. The visible wavelength interval is divided by a dichroic at around $600 \mathrm{~nm}$ feeds two essentially similar spectrographs. Observations are presented, with a 2-pixel resolution of $2 \AA$.

\section{Search of Galactic bulge RCB stars}

We used a new method to identify RCB stars. This method is based on the particular RCB's circumstellar shell brigthness. Tisserand et al. (2009, Fig. 4) illustrated that on average Magellanic RCB shells are fainter at $8 \mu \mathrm{m}$ than those of most common AGB stars, but also cooler than those of classical carbon stars. This can be explained by the highly non-spherical dust distribution around RCB stars as their dust shell is formed from the ejection of dust clouds in random directions (Clayton 1996; de Laverny \& Mékarnia 2004; Wood \& Nicholls 2009). A selection of RCB candidates based on their mid-infrared magnitudes and colours should therefore greatly simplify the search for RCB stars as the number of objects selected for further followup drops dramatically. Effectively, classical methods are based mainly on optical light curve analyses dedicated to identify random fast decreases in brightness. These methods are highly timeconsuming as RCB have to be searched for among millions of light curves. Furthermore, the mid-infrared method is less biased: inactive RCB stars can now be found, as well as very active ones that remain fainter in the optical.

Our search of bulge RCB stars was performed in four consecutive steps. The details and complications of the selection criteria are described below. First, we selected candidates based on broadband mid-infrared magnitudes and colours using the Spitzer GLIMPSE catalogues (II and III) (Churchwell et al. 2009). Second, we rejected all objects not showing a nearinfrared excess in the 2MASS catalogue. Third, we examined at the optical light curves of all remaining objects with a counterpart in the OGLE-II and -III catalogues and selected those objects that exhibit suspicious rapid declines in brightness. Finally, we followed up spectroscopically most of the selected objects.

We note also that we observed spectroscopically a number of optically bright objects selected on the basis only of the two infrared criteria but located outside the OGLE-III fields. A few of them have an atmosphere rich in carbon and are therefore now considered as RCB star candidates. Additional explanations are given in Sect. 3.3.

\subsection{Selection from near-infrared and mid-infrared properties}

The Spitzer GLIMPSE surveys observed the Galactic bulge and plane, and catalogued millions of objects (Churchwell et al. 2009). The published archive catalogues contain four midinfrared broadband magnitudes centred on 3.6, 4.5, 5.8, and $8.0 \mu \mathrm{m}$, obtained with the Spitzer IRAC camera. Despite the short exposure time used (1.2 s), we estimated that bulge RCB stars should be saturated in the first two bands and be at the limit of saturation in the last two ${ }^{2}$. We therefore only used the broadband magnitudes [5.8] and [8.0] to select RCB star candidates. In Table 5, we list the mid-infrared magnitudes of the

\footnotetext{
1 Australian National University.

2 Saturation limit: [3.6 $]_{\mathrm{sat}} \sim 7.0 ;[4.5]_{\mathrm{sat}} \sim 6.5 ;[5.8]_{\mathrm{sat}} \sim 4.0$; $[8.0]_{\text {sat }} \sim 4.0$ (Robitaille et al. 2007).
} 

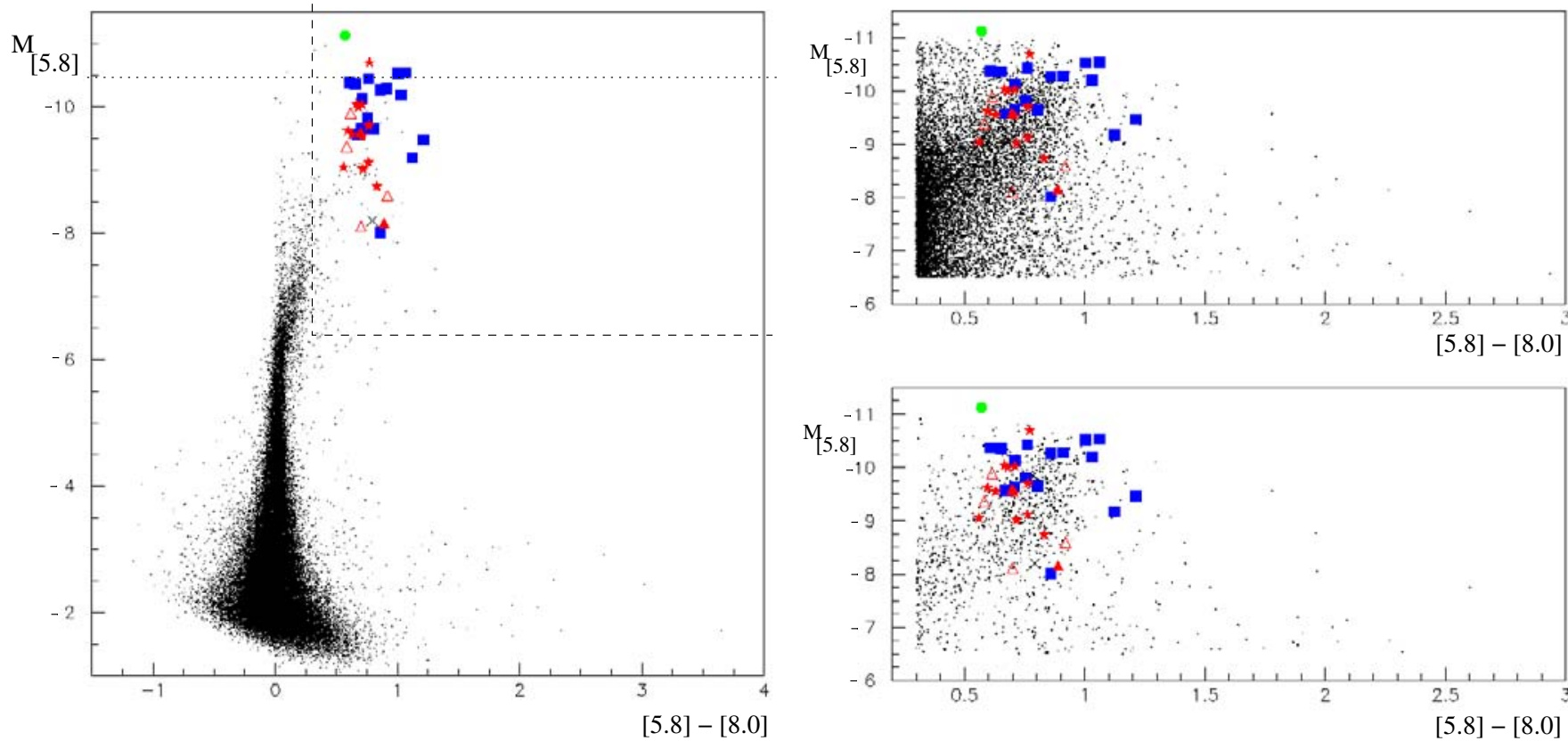

Fig. 1. Left: CMD absolute magnitude $M_{[5.8]}$ versus [5.8]-[8.0]. For the sake of clarity, the dot points represent only all objects catalogued in one $1 \times 2.5 \mathrm{deg}^{2}$ GLIMPSE area centred on $l \sim 0.5, b \sim-3 \mathrm{deg}$. The known RCB stars are represented by larger symbols: blue squares for the Large Magellanic Cloud (Alcock et al. 2001; Tisserand et al. 2009), green circle for the Small Magellanic Cloud (Tisserand et al. 2004), and red stars for the Galactic bulge (Zaniewski et al. 2005; Tisserand et al. 2008). The newly confirmed bulge RCBs are represented by full red triangles and the new candidates by empty red triangles. The RCB candidate KDM 5651 (Morgan et al. 2003) is represented by a cross. We used distance moduli of 18.5, 18.9, and 14.4 for the LMC, SMC, and Galactic center (GC) respectively. The selection area is delimited by dashed lines and the saturation level by a dotted one. Right: same as left, but the dot points represent all objects selected in the entire GLIMPSE area after the first mid-infrared selection (top) and the remaining objects after the second selection on near-infrared excess (bottom).

Table 1. Numbers of objects preselected.

\begin{tabular}{lcccc}
\hline \hline Criteria & 1 & 2 & 3 & Total \\
\hline Entire GLIMPSE-II & 772 & 136 & 1072 & 1980 \\
Entire GLIMPSE-III & 625 & 204 & 299 & 1128 \\
Located in an OGLE-III fields & 154 & 26 & 91 & 271 \\
Catalogued by OGLE-III & 150 & 26 & 90 & 266 \\
Number of Known RCB found & 5 & 1 & 1 & 7 \\
Number of new RCB found & 3 & 0 & 0 & 3 \\
\hline
\end{tabular}

known bulge RCB stars found in the GLIMPSE archive catalogues. We used these archive catalogues because they are more complete and therefore optimise our chance of finding RCB stars that are even saturated. We note that the saturation level depends on the background emission, which experiences strong variation in the GLIMPSE fields. We obtained measurements for all the 13 known bulge RCB stars present in the GLIMPSE fields, but one, EROS2-CG-RCB-1, has no measurement in the [8.0] band. It is also the closest known RCB to the Galactic plane, where the background emission is higher (see Churchwell et al. 2009, for more explanation).

We used three different selection criteria to optimise our chance of finding bulge RCB stars. Each criterion uses the near-infrared 2MASS $J, H$, and $K$ magnitudes, as well as the Spitzer/GLIMPSE [5.8] and [8.0] ones, which are explained hereafter. The first criterion is described in more detail as it corresponds to the favourable case where all magnitude measurements are available. The numbers of selected objects from each selection criterion are listed in Table 1.
- Criterion 1: Figure 1, left side, presents the colour magnitude diagram (CMD), i.e., plot of absolute magnitude $M_{[5.8]}$ versus [5.8] - [8.0], of all objects located in one GLIMPSEIII field and presenting non-null values in [5.8] and [8.0] magnitudes. The known Galactic bulge and Magellanic RCBs are indicated as well as the selection limits and the saturation level. We selected all objects brighter than $M_{[5.8]}<$ -6.5 with a colour index [5.8] - [8.0] redder than 0.3. The selection criteria were based on the known Magellanic and Galactic bulge RCB magnitudes and colours. We can conclude on the basis of resuls for the Magellanic RCBs that most bulge RCBs should not be saturated in the [5.8] band. However, as RCBs have a positive [5.8] - [8.0] colour and the saturation level in these two bands is identical, we expect that some bulge RCBs be saturated in the [8.0] band ${ }^{3}$. This last remark is supported by the almost constant [5.8] - [8.0] colour observed for the bulge RCBs. Finally, we note that there is good overlap between the Magellanic and Galactic bulge RCBs, supporting again a bulge location for these last ones.

The two figures on the right side of Fig. 1 present all selected objects in the GLIMPSE-III fields after the midinfrared selection (top figure) that we have just described and the remaining objects after the near-infrared second selection (bottom one). This near-infrared selection was designed to select all objects displaying a near-infrared excess. The applied near-infrared criteria are shown in Fig. 2, on the left side. We retained only all objects with measure-

\footnotetext{
${ }^{3}$ Note that it should not affect our selection criteria if a [8.0] measurement exists.
} 

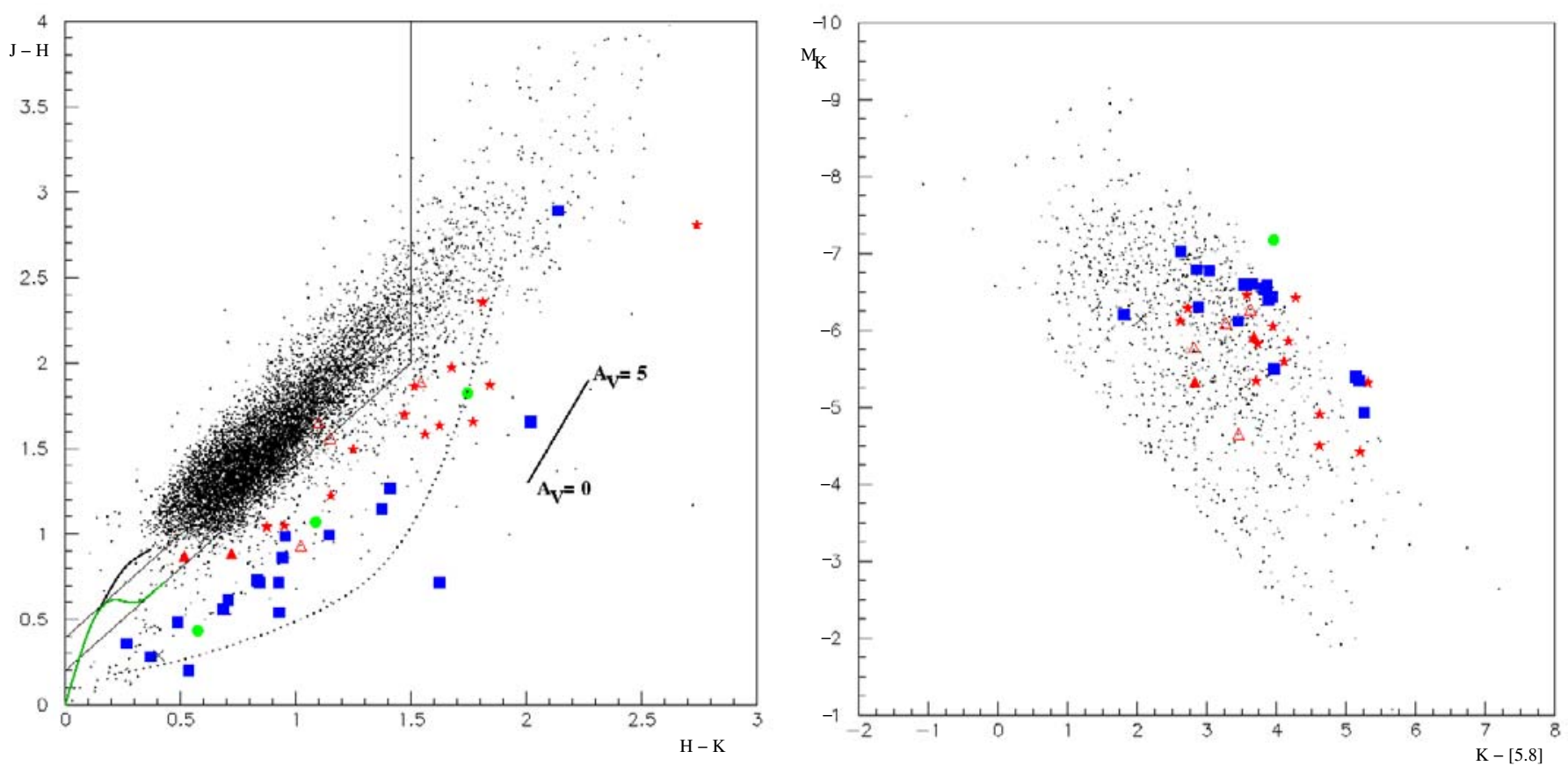

Fig. 2. Left: $J-H$ versus $H-K$ colour diagram. The dot points represent all objects selected with the mid-infrared selection criteria. All the new and known Magellanic and Galactic bulge RCB stars are represented; their symbols are identical to those in Fig. 1. The dotted curve corresponds to the combination of black bodies consisting of a $5500 \mathrm{~K}$ star and a $1000 \mathrm{~K}$ dust shell in various proportions ranging from all "star" to all "shell" (from Feast 1997). The straight lines delimit the near-infrared selection area (see Eqs. (1) and (2)). The line on the right side represents the reddening vector of Rieke \& Lebofsky (1985). Also shown are the expected positions (lines in the bottom-left side) of common dwarf (green) and giant (black) stars from Bessell \& Brett (1988). Right: CMD absolute magnitude $M_{K}$ versus $K-$ [5.8] of all objects that passed the two infrared selection criteria.

ments in all three 2MASS bands that pass the following pragmatic criteria depending on the GLIMPSE survey analysed, GLIMPSE-II (GII) area being more affected by interstellar extinction than GLIMPSE-III (GIII):

$$
\begin{aligned}
\text { GII: } & J-H<1.2(H-K)+0.4 \text { or } H-K>1.5 \\
\text { GIII: } & J-H<1.2(H-K)+0.2 \text { or } H-K>1.5 .
\end{aligned}
$$

We note that this near-infrared selection criteria reject most of the classical carbon stars, distributed along the main locus of Fig. 2, left side. The right-hand side shows the distribution of all remaining stars after both infrared selection criteria in a CMD of absolute magnitude $M_{K}$ vs. $K-$ [5.8]. We interpret the $\sim 0.8$ mag difference between Magellanic and Galactic bulge RCBs as being caused by the interstellar extinction that affects the latter group.

Interestingly, we emphasize that an overdensity appeared after both infrared selections had been applied, in the colour magnitude diagram [5.8] vs. [5.8] - [8.0] (Fig. 1, bottomright) at the expected brightness and temperature of known RCB star shells.

- Criterion 2: The number of RCB stars with no information in the mid-infrared [8.0] band due to saturation, such as EROS-2-CG-RCB-1, may be important. This situation may be more common closer to the Galactic plane as the background emission becomes higher. Therefore, for this second series of criteria, we kept all objects that have no measurement in the [8.0] band but a valid one in [5.8], and selected all bright objects with $M_{[5.8]}<-8.5$. We increased the value of this last selection level in comparison to the first criterion because only really bright RCBs in [5.8] should be saturated in the [8.0] band (RCBs [5.8] - [8.0] colour index values range between 0.5 and 1.5 (see Fig. 1)). Afterward, we applied the same near-infrared selection cuts as presented in criterion 1 (see Eqs. (1) and (2)), except that we added an upper limit to the $\mathrm{K}$ band brightness of $M_{K}<-8$.

- Criterion 3: RCB stars could have been observed by 2MASS during an obscured phase caused by dust clouds. Therefore, we can expect to find RCB stars with missing information in near-infrared but consistent values in the mid-infrared. This problem should also be more common at lower latitude as the interstellar extinction becomes higher. We kept all objects with a valid measurement in $K$, [5.8], and [8.0] but missing one in $J$ and/or $H$. We applied the same mid-infrared selection as for criterion 1 and added two further limits related to the $K$ band of $M_{K}<-8$ and $K-[5.8]>2.0$. We note that with an $M_{K}$ absolute magnitude of about -6, RCB stars are almost always visible in that particular band towards the bulge: even in the case of an extreme extinction, $A_{V} \sim 30$, bulge RCB stars would still have a $K$ magnitude of $\sim 11.4$ $\left(A_{K} \sim 0.1 A_{V}\right)$.

Seven previously known RCB stars were in our fields and should have been discovered using the selection criteria that we have just explained. They were all catalogued by both Spitzer/GLIMPSE and OGLE-III surveys and we list them in Table 2. We successfully found all of these 7 RCB stars. We note that they would all have been considered directly as RCB stars because they exhibit sharp declines in their respective OGLE light curve . $^{4}$

${ }^{4}$ The OGLE-III light curves of all known RCB stars are available at the following URL: http://ogle.astrouw.edu.pl/ogle3/rcom/ rcom.html. This web site present a useful real-time monitoring of known RCBs (Udalski 2008). 


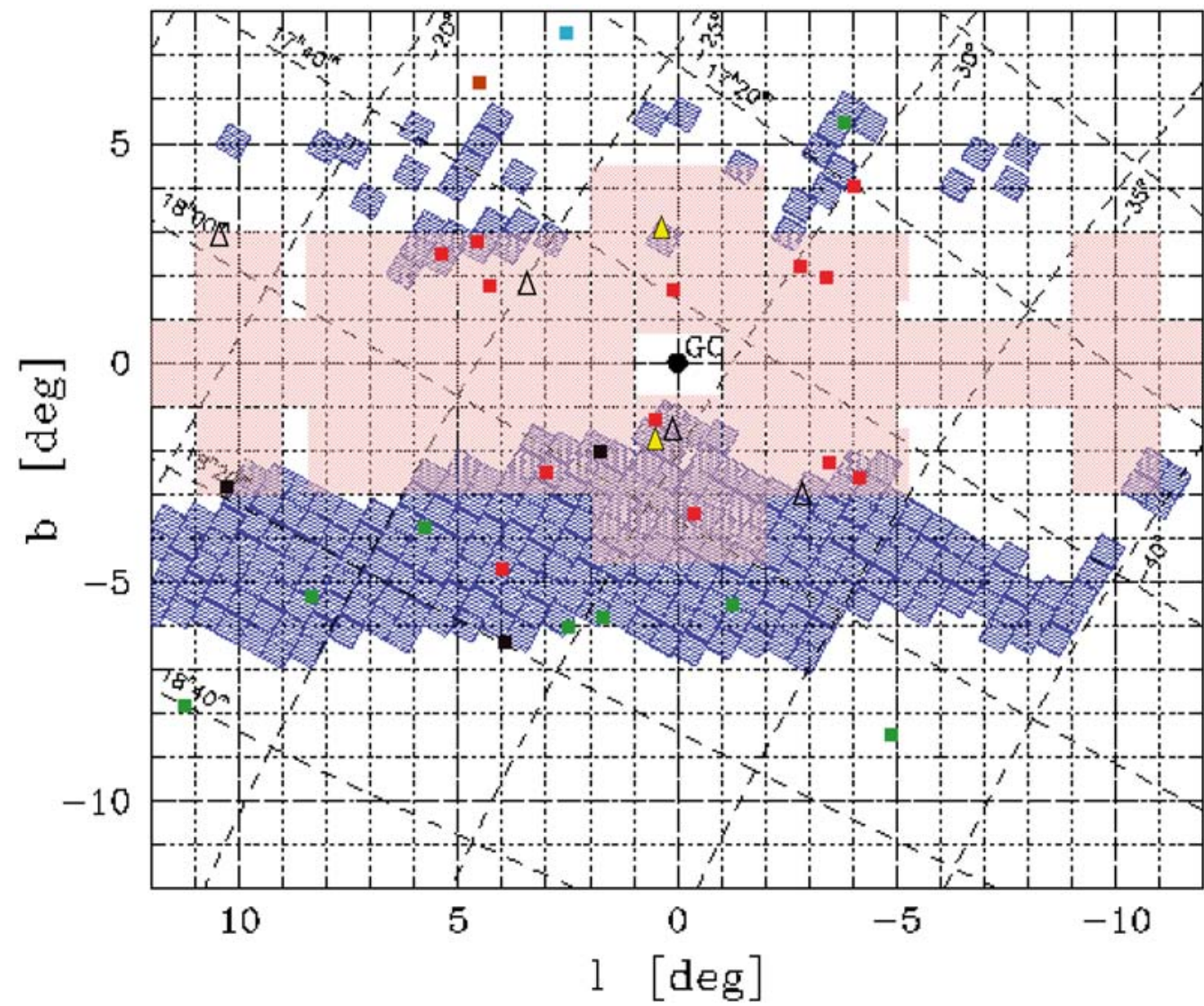
- Clayton, 1996
= V2552 Oph, Hesselbach et al. 2003
- Zaniewski et al., 2005
- Tisserand et al., 2008
- V532 Oph, Clayton et al. 2009
$\triangle$ New OGLE confirmed RCBs
$\triangle$ New RCB candidates

OGLE-III fields

GLIMPSE fields

Fig. 3. Galactic bulge map with a representation of the area observed by the Spitzer/GLIMPSE survey and the fields monitored by the OGLEIII survey. The location of the newly confirmed and candidate Galactic RCBs stars are indicated along with 13 discovered inside the bulge by Tisserand et al. (2008), four reported by Zaniewski et al. (2005), the newly active warm RCB V 2552 Oph (Hesselbach et al. 2003), V 532 Oph (Clayton et al. 2009), and the other previously known RCBs in this region (V1783 Sgr, V739 Sgr, V3795 Sgr, VZ Sgr, V517 Oph, Gu Sgr, V348 Sgr, and WX Cra).

Table 2. Known Galactic RCB stars located in the OGLE-III fields.

\begin{tabular}{lcc}
\hline \hline RCB name & OGLE-III id. & $\begin{array}{c}\text { Located in a } \\
\text { GLIMPSE field? }\end{array}$ \\
\hline EROS2-CG-RCB-1 & BLG195.5.23 & yes \\
EROS2-CG-RCB-3 & BLG182.1.96290 & yes \\
EROS2-CG-RCB-5 & BLG138.1.130117 & yes \\
EROS2-CG-RCB-10 & BLG345.6.159428 & yes \\
EROS2-CG-RCB-11 & BLG343.6.122070 & yes \\
EROS2-CG-RCB-13 & BLG224.3.130650 & yes \\
EROS2-CG-RCB-14 & BLG228.2.23975 & no \\
MACHO-135.27132.51 & BLG213.4.129296 & no \\
MACHO-401.48170.2237 & BLG214.2.215079 & yes \\
V 517 Oph & $\mathrm{x}$ & no \\
V 1783 Sgr & BLG160.8.4580 & no \\
V 739 Sgr & BLG193.1.103298 & no \\
V 3795 Sgr & BLG252.6.166190 & no \\
VZ Sgr & x & no \\
GU Sgr & BLG275.2.39337 & no \\
\hline
\end{tabular}

Notes. x: Not catalogued as too bright on template image.

\subsection{OGLE light curves}

Among all selected objects in the GLIMPSE fields, we inspected visually the light curves of the ones catalogued in the OGLE-II and OGLE-III fields. The overlap between the GLIMPSE area and the OGLE-III fields is represented in Fig. 3 (Note that the area covered by the OGLE-III fields include the OGLE-II ones).
Of the 271 candidates located in the OGLE-III fields, 266 were catalogued. Four of the remaining ones were saturated in the reference image and the other one was too faint ${ }^{5}$. The light curve visual inspection left us with no doubt about the RCB nature of two objects, named OGLE-GC-RCB-1 and OGLE-GC-RCB-2. Their coordinates are presented in Table 3, light curves in Figs. 5 and 6, and charts in Fig. 7. OGLE-GC-RCB-1 exhibits six consecutive drops from 4 to up to 9 mag in its OGLE-II and -III light curves, while OGLE-GC-RCB-2 has multiple rapid and spectacular declines, up to $8 \mathrm{mag}$, immediately followed by rapid recoveries. Overall, among the 266 stars, 20 stars were selected during the visual inspection process: 10 were selected due to non-periodic and sudden declines present in their light curves, and another 10 were selected because their light curves resemble RCB star behaviour during a recovery or a bright phase. We followed-up these 20 stars spectroscopically.

\subsection{Spectra}

If a well-sampled light curve is available, identification with the $\mathrm{RCB}$ class can be made with fairly high confidence because of the distinct nature of the RCB brightness drops (fast and up to $9 \mathrm{mag}$ ). However, if these declines remain small, spectroscopic information is necessary to reveal and confirm their true nature.

\footnotetext{
5 We note that this particular star $(\mathrm{RA}=18: 03: 20.368$, Dec $=$ $-27: 32: 24.27)$ was catalogued in the USNO-B1.0 catalogue in 1982.4 as a bright star: $R 2 \sim 13.68$.
} 
Table 3. General information about the newly confirmed and candidate RCB stars.

\begin{tabular}{|c|c|c|c|c|}
\hline RCB name & $\begin{array}{l}\text { OGLE-III } \\
\text { identifier }\end{array}$ & $\begin{array}{l}\text { OGLE-II } \\
\text { identifier }\end{array}$ & Coordinates $\left(\mathrm{J}_{2000}\right)$ & Other identifier \\
\hline OGLE-GC-RCB-1 & BLG333.5.145097 & $\begin{array}{l}\text { New RCB stars } \\
\text { BUL_SC43.326584 }\end{array}$ & $17: 35: 18.125-26: 53: 49.17$ & $\begin{array}{l}\text { NSV 22773; Terz } 2637 \\
\text { IRAS 17321-2652 }\end{array}$ \\
\hline OGLE-GC-RCB-2 & $\begin{array}{c}\text { BLG101.4.230792 } \\
\text { BLG195.1.8088 }\end{array}$ & BUL_SC3.793780 & 17:53:57.078 -29:31:42.90 & $\begin{array}{c}\text { IRAS 17507-2931 } \\
\text { ASAS_175357-2931.8 }\end{array}$ \\
\hline \multicolumn{5}{|c|}{ New RCB star candidates } \\
\hline OGLE-GC-RCB-Cand-1 & BLG194.1.90513 & BUL_SC37.133492 & $17: 52: 14.926-29: 38: 30.47$ & EROS2-cg0034111292 \\
\hline OGLE-GC-RCB-Cand-2 & $\begin{array}{c}\text { BLG148.5.148667 } \\
\text { BLG155.8.10916 }\end{array}$ & & 17:51:19.507 -32:43:21.44 & EROS2-cg0766k15255 \\
\hline GLIMPSE-RCB-Cand-1 & & & $17: 46: 45.916-25: 03: 14.05$ & \\
\hline GLIMPSE-RCB-Cand-2 & & & $17: 57: 49.999-18: 25: 22.89$ & \\
\hline
\end{tabular}

Table 4. Near-IR photometry.

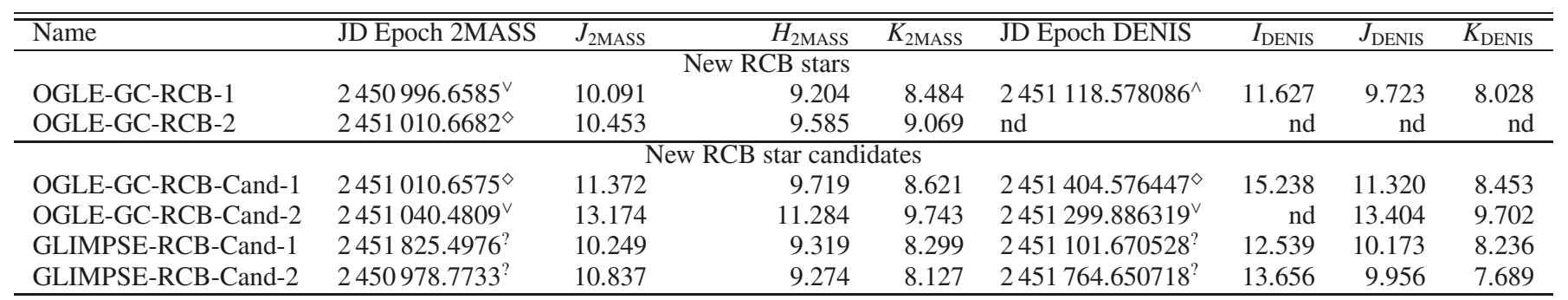

Notes. ${ }^{\diamond}$ During a bright phase, ${ }^{\vee}$ and ${ }^{\wedge}$ during a dimming or recovering phase, $?$ phase unknown.

Most of the 20 stars selected for spectroscopy follow-up, were found to be either $\mathrm{M}$ giant stars exhibiting strong TiO bands features in their spectra, or hot stars presenting emission lines for half of them. However one star, named hereafter OGLE-GCRCB-1, exhibits strong carbon features in its spectrum and can be assumed to be a new confirmed RCB stars. More discussion about this star is presented in Sect. 4. Unfortunately, we did not obtain spectra for OGLE-GC-RCB-2 and another star considered to be a good RCB candidate and named hereafter OGLEGC-RCB-Cand-1 in Table 3. The carbon spectra obtained are presented in Fig. 4.

We also decided to target spectroscopically some optically bright objects selected by one of the 3 infrared criteria, but located outside the OGLE-III fields, to estimate the efficiency of finding RCB stars during a blind survey (i.e without light curves being available). Among the 11 randomly selected GLIMPSE objects, two display an atmosphere rich in carbon (see Fig. 4) and are therefore now considered as RCB star candidates, they are named GLIMPSE-RCB-Cand-1 and - 2 in Table 3.

From a simple analysis of the spectra, we can empirically compare the different temperatures of the newly confirmed and candidate RCB stars, based on the strength of their Ca II triplet absorption lines. The intensity of these lines is, as shown by Richer (1971), a good indicator of carbon star temperature: the cooler the temperature, the weaker the lines. The RCB candidate GLIMPSE-RCB-Cand-1 clearly seems to be hotter than the other 3 carbon stars observed and the candidate OGLE-GCRCB-Cand-2, the coolest. We also searched for the isotope ${ }^{13} \mathrm{C}$ in the atmosphere of these stars. We used the spectral atlas of carbon stars compiled by Barnbaum et al. (1996) to identify isotopic $\mathrm{C}_{2}$ and $\mathrm{CN}$ bands, respectively at 6100 and $6260 \AA$. We found no trace of ${ }^{13} \mathrm{C}$ in OGLE-GC-RCB-1 and the two candidates GLIMPSE-RCB-Cand-1 and -2 , but a positive signature for OGLE-GC-RCB-Cand-2. Indeed, a bandhead feature due to
${ }^{13} \mathrm{CN}$ is recognisable at $\sim 6260 \AA$, even if the signal is low in that particular wavelength range.

\section{Discussion about new RCB stars}

The newly confirmed and candidate RCB stars are listed in Table 3, and their near- and mid- infrared photometry in Tables 4 and 5. Their light curves and charts are presented, respectively, in Figs. 5 and 7. We note interestingly that we found an entry for most of the known and new RCB stars in the MSX (Midcourse Space Experiment) point source catalogue (Egan et al. 2003). MSX magnitudes are also listed in Table 5. The MSX survey has observed the entire Galactic plane, with $|b| \leqslant 6 \mathrm{deg}$, in four different bands: $A(8.26 \mu \mathrm{m}), C(12.12 \mu \mathrm{m}), D(14.65 \mu \mathrm{m})$, and $E(21.41 \mu \mathrm{m})$. Only objects brighter than $A \lesssim 8$ mag were detected, the A band being the most sensitive. We emphasize that MSX magnitudes could help in the future to select new RCB candidates.

In Table 6, we present $V$ and $I$ maximum magnitudes of the 4 OGLE RCBs, confirmed and candidates. Those magnitudes were estimated from their respective OGLE-II and -III light curves. We applied an interstellar extinction and reddening correction to these magnitudes using the same technique applied in Tisserand et al. (2008), where an average extinction $A_{\mathrm{Be}}{ }^{6}$ was calculated using red clump giant stars located around each RCB. We therefore obtained the extinction-corrected RCBs magnitude $V_{0}$ and colour $(V-I)_{0}$ at maximum brightness. Absolute magnitudes $M_{V}$ were calculated using a bulge distance moduli of $14.4 \mathrm{mag}$. The values presented in Table 6 can be compared to the $M_{V}$ vs. $(V-I)_{0}$ colour magnitude diagram presented in Tisserand et al. (2008, Fig. 7): one can see that OGLE-GC-RCB-2 is a rare warm RCB star, as warm as W Men in the LMC; OGLE-GC-RCB-1 is a more classical RCB with a

${ }^{6}$ Be corresponds to the blue EROS2 filter, see Tisserand et al. (2007). 

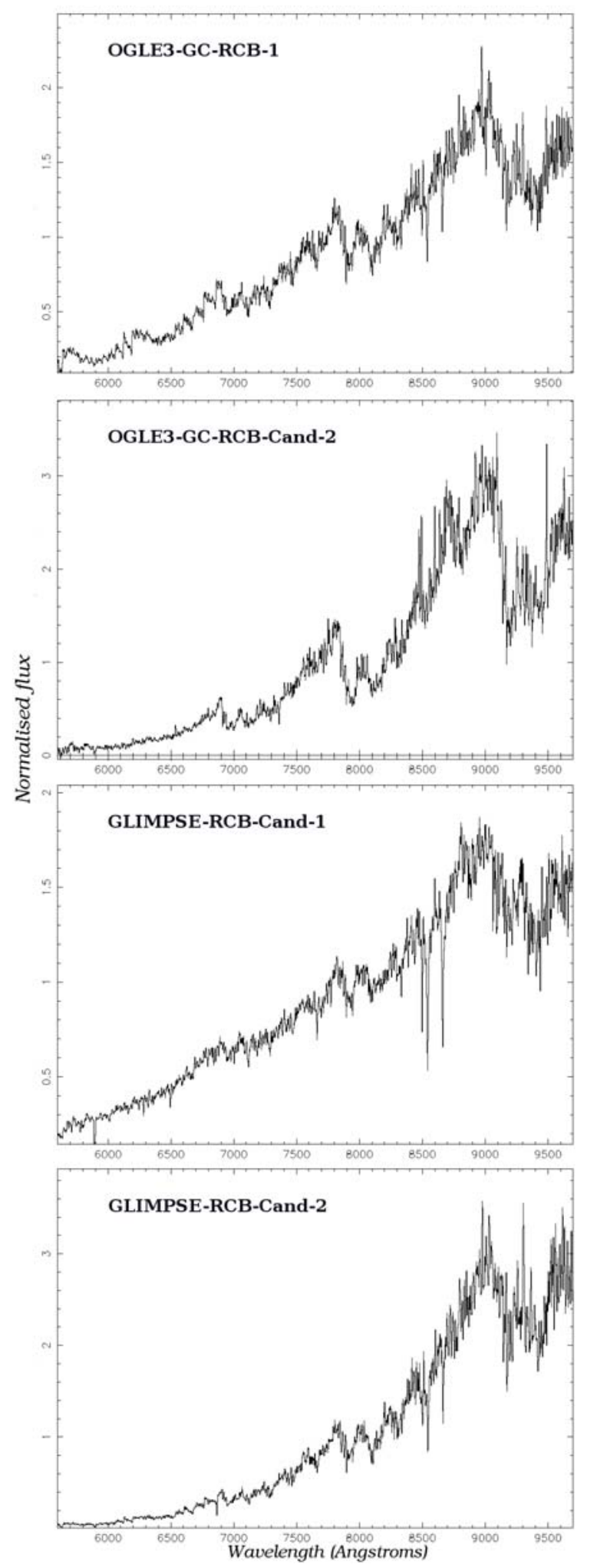

Fig. 4. Spectra of the new confirmed and candidates RCB stars. The flux $\left(F_{\lambda}\right)$ is normalised to the flux at $8000 \AA$.

temperature around $T_{\text {eff }} \sim 5000 \mathrm{~K}$; and OGLE-GC-RCB-Cand-1 and -2 would be extremely cool objects in the RCB class.

We discuss now each new RCB star. We note that none of them have been catalogued in the MACHO database, but two,

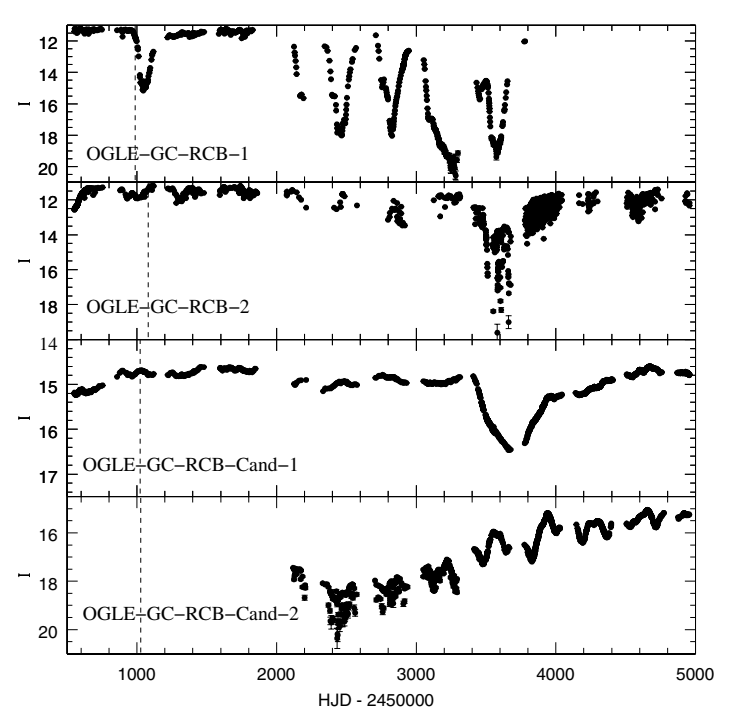

Fig. 5. OGLE-II and OGLE-III light curves of the new RCB stars, confirmed and candidates. The dashed vertical lines indicate the 2MASS epochs.

candidates OGLE-GC-RCB-Cand-1 and -2, were monitored by the EROS-2 survey ${ }^{7}$.

- OGLE-GC-RCB-1: OGLE-II and -III light curves show that this RCB star has recently experienced a high level of dust production activity as many declines from 4 and up to 9 mag are observable. It was named Terz V 2637 by Terzan \& Gosset (1991) due to a four magnitude variation observed in 1987 between two $R$ band epochs separated by 1 month. We also note that it was catalogued as a possible RCB star in the AAVSO International Variable Star Index (Watson 2006). With its numerous significant and rapid optical declines, bright shell, carbon spectrum with absence of ${ }^{13} \mathrm{C}$ in it, OGLE-GC-RCB-1 is clearly a typical RCB star with a temperature around $T_{\text {eff }} \sim 5000 \mathrm{~K}$.

- OGLE-GC-RCB-2 has undergone a 350 days long phase where multiple rapid and spectacular declines, up to 8 mag, were immediately followed by rapid recoveries. We did not obtain any spectrum of this star, but its light curve and infrared emission indicate that OGLE-GC-RCB-2 is an RCB star. It was bright enough to be monitored by the ASAS-3 survey $^{8}$. We underline that OGLE-GC-RCB-2 exhibits the faintest shell of any RCB star. Its mid-infrared brightness is similar to those of both the warm LMC RCB star W Men and the cool LMC RCB candidate KDM-5651 (Morgan et al. 2003). This indicates that OGLE-GC-RCB-2 is in a low ejection activity phase. OGLE-GC-RCB-2 is the warmest RCB star known in the bulge, with an absolute magnitude and intrinsic $(V-I)_{0}$ colour similar to W Men (see Table 6 and Tisserand et al. 2008, Fig. 7). We note that the temperature of W Men was estimated at $T_{\text {eff }} \sim 7000 \mathrm{~K}$ by Goldsmith et al. (1990).

- RCB candidate OGLE-GC-RCB-Cand-1 exhibits only a slow 1.6 mag decline in its OGLE light curve, with a decline rate of $\sim 0.006 \mathrm{mag} / \mathrm{day}$ followed by a relatively

7 OGLE-GC-RCB-1 and OGLE-GC-RCB-2 were saturated on the EROS2 reference images.

8 ASAS: All Sky Automated Survey (Pojmanski 1997), URL: http://www . astrouw . edu.pl/asas $/$ ?page=main 
Table 5. Spitzer/GLIMPSE II and III and MSX magnitudes for bulge RCB stars.

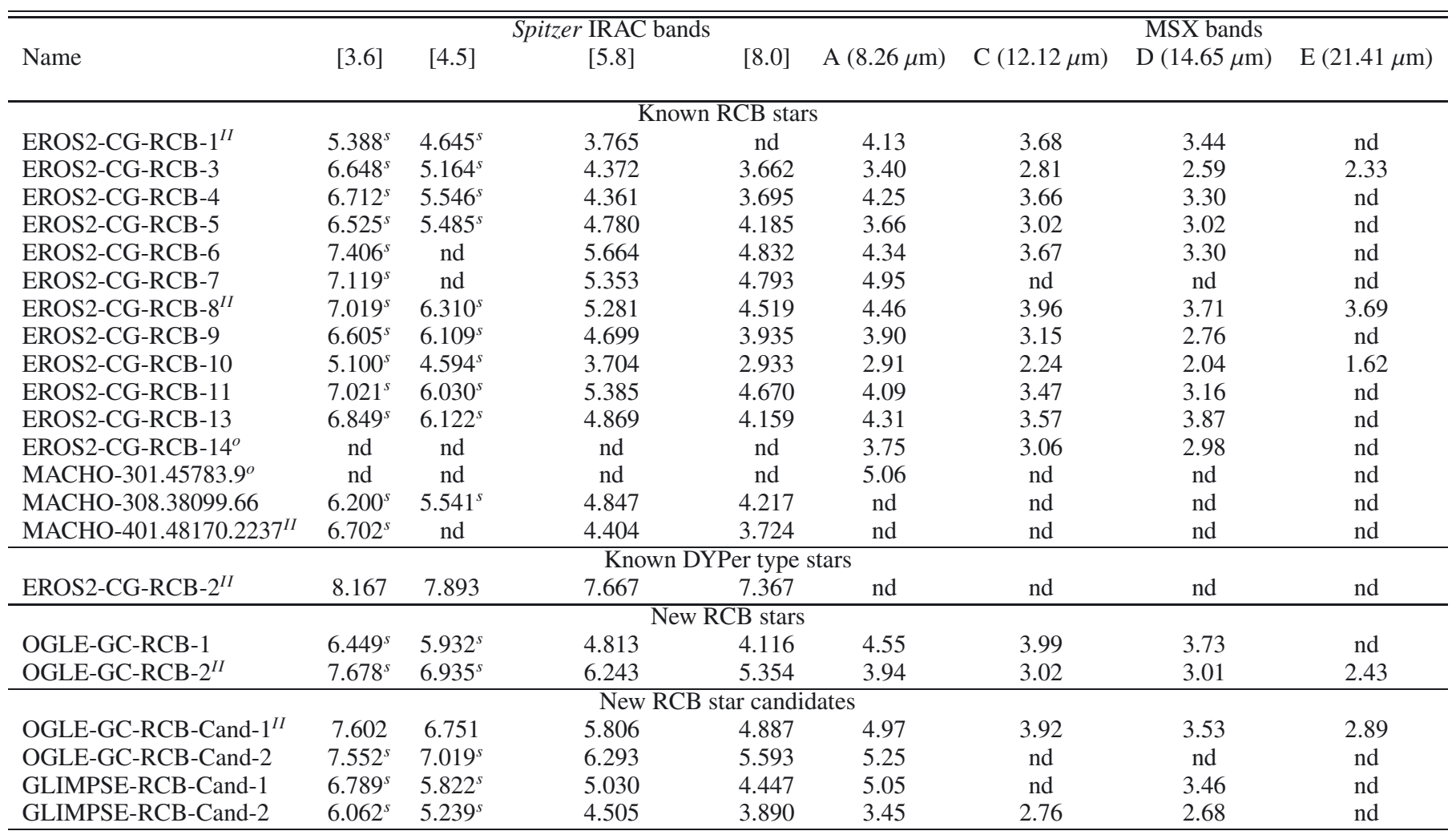

Notes. nd = not detected; ${ }^{s}$ probably saturated, measurement can't be trusted. ${ }^{I I}$ Spitzer magnitudes from GLIMPSE-II-Archive catalogues, instead of GLIMPSE-III-Archive for the others. ${ }^{\circ}$ Located outside Spitzer GLIMPSE fields.

Table 6. Magnitudes $I_{\max }$ and colours $(V-I)_{\max }$ of the new OGLE Galactic RCBs at maximum brightness, with derived absolute magnitudes $M_{V}$ and intrinsec colours $(V-I)_{0}$ after extinction correction.

\begin{tabular}{lcccccc}
\hline \hline OGLE RCBs & $(V-I)_{\max }$ & $I_{\max }$ & $A_{\mathrm{Be}}$ & $V_{0}$ & $(V-I)_{0}$ & $M_{V}$ \\
\hline RCB-1 & 3.20 & 11.30 & 2.96 & 10.87 & 1.53 & -3.53 \\
RCB-2 & 2.10 & 11.50 & 3.06 & 9.85 & 0.22 & -4.55 \\
Cand-1 & 5.20 & 14.70 & 3.53 & 15.57 & 3.20 & 1.17 \\
Cand-2 & 5.20 & 15.20 & 3.09 & 16.61 & 3.46 & 2.21 \\
\hline
\end{tabular}

symmetric immediate recovery, resembling those observed in DY Per type of stars (see Tisserand et al. 2009, for some examples). This last remark is also supported by its position in the $J-H$ versus $H-K$ colour-colour diagram (Fig. 2), close to the expected locus of classical carbon stars. However we note that OGLE-GC-RCB-Cand-1 was catalogued in the USNO-B1.0 catalogue with a faint magnitude of $R$ 19.4 mag observed in 1986.4 (Monet et al. 2003), which is about 4 mag fainter than the maximum brightness observed by OGLE. Therefore, OGLE-GC-RCB-Cand-1 should still be considered as an RCB candidate. Unfortunatly, we did not obtain any spectrum of that star. From its derived intrinsic $(V-I)_{0}$ colour, OGLE-GC-RCB-Cand-1 is a cool RCB star candidate.

- RCB candidate OGLE-GC-RCB-Cand-2 is a special case. Indeed, its OGLE-III light curve diplays a slow recovery phase with large oscillations following a slow decline that lasted more than 2000 days. This slow decline phase is observable in the EROS-2 light curve (Fig. 6). On top of the slow decline, one can observe a fast drop of about 2 mag

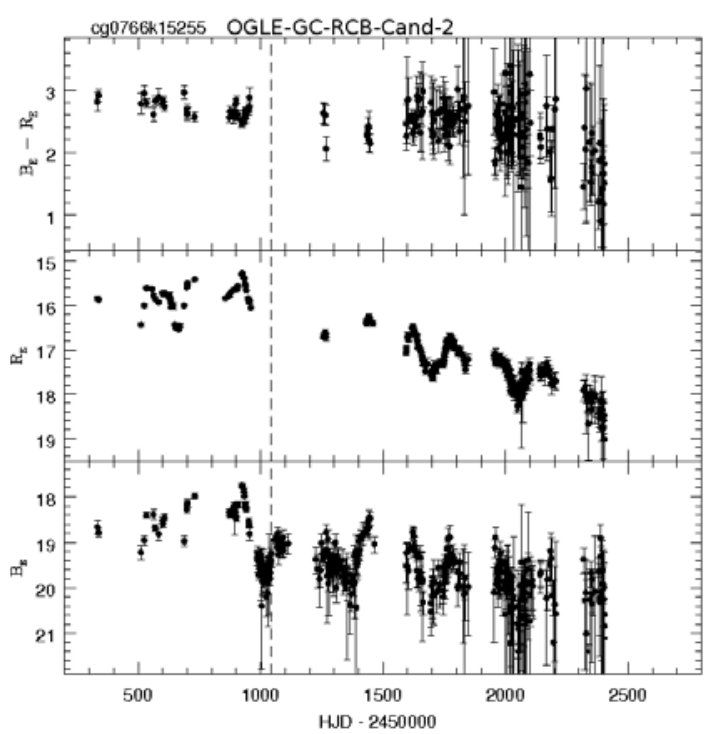

Fig. 6. EROS-2 light curve of candidate OGLE-GC-RCB-Cand-2; top: $B_{E}-R_{E}$ colour versus time; middle: $R_{E}$ light curve; bottom: $B_{E}$ light curve. The dashed vertical lines indicate the 2 MASS epochs.

(around HJD 930) with a decline rate of $\sim 0.026 \mathrm{mag} / \mathrm{day}$. This uncommon optical behaviour resembles that of the RCB star EROS2-LMC-RCB-2 (Tisserand et al. 2009) where a slow decline and recovery were also observed ${ }^{9}$. We note that

\footnotetext{
9 We note that such declines were also observed in the 200 years light curve of R CrB.
} 


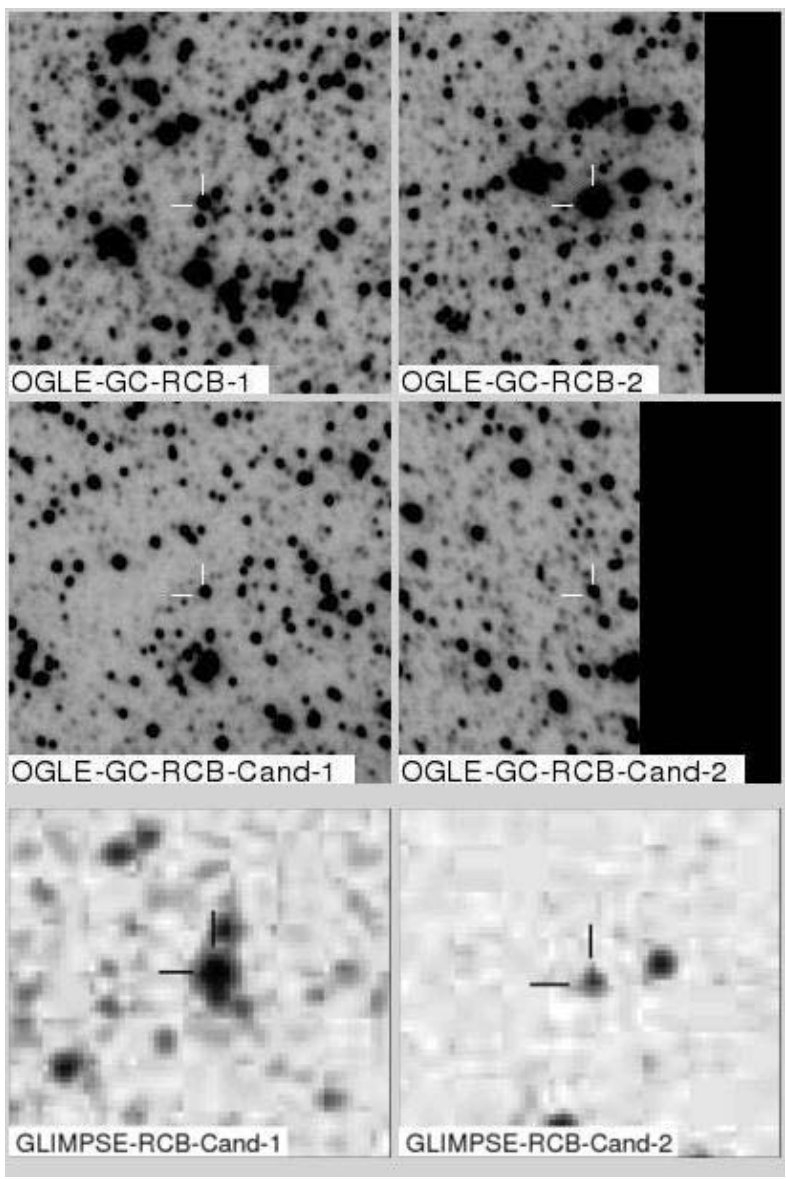

Fig. 7. Charts of the newly confirmed and candidate RCB stars $\left(1^{\prime} \times 1^{\prime}\right)$. North is up, east is to the left. The top images are from the OGLE-III survey, the bottom ones from USNO.

OGLE-GC-RCB-Cand-2 seems to be a very cool carbon star because its spectrum displays weak Ca II triplet absorption lines. This is consistent with its very red $(V-I)_{0}$ colour (see Table 6). With this $(V-I)_{0}$ colour and absolute magnitude $M_{V}$, OGLE-GC-RCB-Cand-2 may indeed correspond to the faintest and coolest RCB star (see Tisserand et al. 2008, Fig. 7). However, its derived magnitudes should not be fully trusted as we did not observe with certainty its maximum magnitude. The time spent at maximum brightness during the OGLE-III and EROS-2 observations was short. The low decline rate observed and its relatively faint shell imply that OGLE-GC-RCB-Cand-2 represents a possible link between RCB stars and DY Per type of stars. This last remark is also supported by the non-negligable presence of ${ }^{13} \mathrm{C}$ in its atmosphere.

- RCB candidates GLIMPSE-RCB-Cand-1 and -2 were both found by a spectroscopic follow-up of optically bright objects $(R \sim 14$ and $\sim 17$, respectively) randomly selected on the basis of their near- and mid- infrared emission. There is no optical light curve available. Both stars have a carbonrich atmosphere and near- and mid- infrared emissions that are typical of RCB stars. We note that we do not detect any traces of ${ }^{13} \mathrm{C}$ features in their spectra, and from the strength of its Ca II triplet absorption lines, GLIMPSE-RCB-Cand-1 is a candidate to be a warm RCB star. For GLIMPSE-RCBCand-2, we note a difference of $\sim 0.9$ and $\sim 0.4$ mag between the two epochs of Denis and 2MASS in the $J$ and $K$ bands, respectively, taken $\sim 786$ days apart. This difference seems to indicate that GLIMPSE-RCB-Cand-2 was in an obscured phase during the Denis epoch. Furthermore, we note that GLIMPSE-RCB-Cand-2 is clearly a variable star, as it appears relatively bright on the USNO SERC photographic plate (see its chart, Fig. 7), and is not visible on the USNO POSS I picture.

\section{Summary}

We have tested new RCB selection criteria to reduce the number of preselected objects for subsequent visual inspection of OGLE light curves prior to spectroscopy follow-up. Thanks to the midinfrared Spitzer/GLIMPSE and near-infrared 2MASS databases, we have selected 266 stars located in both the Spitzer/GLIMPSE and OGLE-III fields. The analysis has led to the discovery of 2 new RCB stars and 4 new candidates. We have demonstrated that we do not need to use the laborious and time-consuming technique of light curve analysis to find new RCB stars. Furthermore, we rediscovered all of the 7 known RCB stars that were located in both the Spitzer/GLIMPSE and OGLE-III monitored area. This indicates the high efficiency of our analysis.

This new technique can now be used to find RCB stars in areas not monitored optically. RCB star searches can therefore be more easily applied to the entire bulge area, even at low Galactic latitude where interstellar extinction is high. We tested this idea by observing spectroscopically 11 optically bright stars randomly selected on the basis of their mid- and near- infrared emissions and located outside any microlensing survey field. We found that 2 stars exhibit signs of an atmosphere rich in carbon, which may now both be considered as RCB candidates. We emphasize that our chances of finding classical carbon rich stars with our analysis is low as the Galactic bulge is known to have a low density of classical carbon stars, if any (Blanco \& McCarthy 1983), and our near and mid - infrared selection eliminates the vast majority of classical carbon stars.

Acknowledgements. We thank Tony Martin-Jones and the anonymous referee for their careful reading and comments. We also thank Mike Bessell and Peter Wood for their expertise concerning the spectroscopic data reduction. This work is based in part on observations made with the Spitzer Space Telescope, which is operated by the Jet Propulsion Laboratory, California Institute of Technology under a contract with NASA. The OGLE project is partially supported by the Polish MNiSW grant N20303032/4275. This publication makes use of data products from the Two Micron All Sky Survey, which is a joint project of the University of Massachusetts and the Infrared Processing and Analysis Centre, California Institute of Technology, funded by the National Aeronautics and Space Administration and the National Science Foundation. The DENIS data have also been used. DENIS is the result of a joint effort involving human and financial contributions of several Institutes mostly located in Europe. It has been supported financially mainly by the French Institut National des Sciences de l'Univers, CNRS, and French Education Ministry, the European Southern Observatory, the State of Baden-Wuerttemberg, and the European Commission under networks of the SCIENCE and Human Capital and Mobility programs, the Landessternwarte, Heidelberg and Institut d'Astrophysique de Paris.

\section{References}

Alcock, C., Allsman, R. A., Alves, D. R., et al. 2001, ApJ, 554, 298 Barnbaum, C., Stone, R. P. S., \& Keenan, P. C. 1996, ApJS, 105, 419

Belczynski, K., Bulik, T., \& Ruiter, A. J. 2005, ApJ, 629, 915

Bessell, M. S., \& Brett, J. M. 1988, PASP, 100, 1134

Blanco, V. M., \& McCarthy, M. F. 1983, AJ, 88, 1442

Churchwell, E., Babler, B. L., Meade, M. R., et al. 2009, PASP, 121, 213

Clayton, G. C. 1996, PASP, 108, 225

Clayton, G. C., Geballe, T. R., Herwig, F., Fryer, C., \& Asplund, M. 2007, ApJ, 662, 1220

Clayton, G. C., Kilkenny, D., Wils, P., \& Welch, D. L. 2009, PASP, 121, 461

de Laverny, P., \& Mékarnia, D. 2004, A\&A, 428, L13

Diehl, S., Fryer, C. L., Hungerford, A., et al. 2008 [arXiv: 0811.4646] 
Dopita, M., Hart, J., McGregor, P., et al. 2007, Ap\&SS, 310, 255

Egan, M. P., Price, S. D., Kraemer, K. E., et al. 2003, VizieR Online Data Catalog, 5114, 0

Fazio, G. G., Hora, J. L., Allen, L. E., et al. 2004, ApJS, 154, 10

Feast, M. W. 1997, MNRAS, 285, 339

Fryer, C. L., \& Diehl, S. 2008, in Hydrogen-Deficient Stars, ed. A. Werner, \& T. Rauch, ASP Conf. Ser., 391, 335

Goldsmith, M. J., Evans, A., Albinson, J. S., \& Bode, M. F. 1990, MNRAS, 245, 119

Hamadache, C., Le Guillou, L., Tisserand, P., et al. 2006, A\&A, 454, 185

Hesselbach, E., Clayton, G. C., \& Smith, P. S. 2003, PASP, 115, 1301

Monet, D. G., Levine, S. E., Canzian, B., et al. 2003, AJ, 125, 984

Morgan, D. H., Hatzidimitriou, D., Cannon, R. D., \& Croke, B. F. W. 2003, MNRAS, 344, 325

Nelemans, G., Yungelson, L. R., Portegies Zwart, S. F., \& Verbunt, F. 2001, A\&A, 365,491

Pandey, G., Lambert, D. L., \& Rao, N. K. 2008, ApJ, 674, 1068

Pojmanski, G. 1997, Acta Astron., 47, 467

Richer, H. B. 1971, ApJ, 167, 521

Rieke, G. H., \& Lebofsky, M. J. 1985, ApJ, 288, 618
Robitaille, T. P. Cohen, M. Whitney, B. A., et al. 2007, AJ, 134, 2099

Ruiter, A. J., Belczynski, K., \& Fryer, C. 2009, ApJ, 699, 2026

Saio, H., \& Jeffery, C. S. 2002, MNRAS, 333, 121

Skrutskie, M. F., Cutri, R. M., Stiening, R., et al. 2006, AJ, 131, 1163

Soszyński, I., Udalski, A., Szymański, M. K., et al. 2009, Acta Astron., 59, 335

Sumi, T., Woźniak, P. R., Udalski, A., et al. 2006, ApJ, 636, 240

Szymanski, M. K. 2005, Acta Astron., 55, 43

Terzan, A., \& Gosset, E. 1991, A\&AS, 90, 451

Tisserand, P., Marquette, J. B., Beaulieu, J. P., et al. 2004, A\&A, 424, 245

Tisserand, P., Le Guillou, L., \& the EROS-2 Coll. 2007, A\&A, 469, 387

Tisserand, P., Marquette, J. B., Wood, P. R., et al. 2008, A\&A, 481, 673

Tisserand, P., Wood, P. R., Marquette, J. B., et al. 2009, A\&A, 501, 985

Udalski, A. 2003, Acta Astron., 53, 291

Udalski, A. 2008, Acta Astron., 58, 187

Watson, C. L. 2006, Soc. Astron. Sci. Annu. Symp., 25, 47

Webbink, R. F. 1984, ApJ, 277, 355

Wood, P. R., \& Nicholls, C. P. 2009, ApJ, 707, 573

Wyrzykowski, Ł., Kozłowski, S., Skowron, J., et al. 2009, MNRAS, 397, 1228

Zaniewski, A., Clayton, G. C., Welch, D. L., et al. 2005, AJ, 130, 2293 